\title{
聚合物 S 形脊波导的优化设计
}

陆荣国, 廖进昆, 李和平, 唐雄贵, 刘永智

电子科技大学光电信息学院, 成都 610054

E-mail: lurongguo@uestc.edu.cn

2009-01-07 收稿, 2009-03-13 接受

总装预研、国家自然科学基金(批准号: 60736038)、国家高技术研究发展计划(编号: 2007AA01Z269)资助项目

摘要 首先利用变分有效折射率法对聚合物脊形多模光波导基模和高阶模的色散特性与横向 场分布进行分析, 研究了波导结构参数对色散特性的影响, 计算出 TM 基模和高阶模的光场分 布, 得出了聚合物脊形光波导的单模传输条件. 然后利用广角有限差分束传播法和有效折射率 法研究了 $\mathrm{S}$ 形脊波导圆弧曲率半径与弯曲损耗的关系, 得出了如下结论: 当圆弧曲率半径大于 $5000 \mu \mathrm{m}$ 后, 即使波导曲率半径增大, 波导的弯曲辐射损耗已不会再明显减小, 光已可以在 $\mathrm{S}$ 形脊波导中稳定地传播了.

关键词

电光聚合物

$\mathrm{S}$ 形脊波导

有效折射率法

变分法

广角有限差分束

传播法
有机聚合物是制作集成光波导器件很有前景的 材料, 其本身所具有的易集成、响应快速、成本低廉 等一系列优点使得有机聚合物光波导成为近年来研 究的热点 ${ }^{[1 \sim 4}$. 同传统的无机光波导材料相比, 有机 聚合物光波导材料有以下优点: (1)在红外至毫米波 段, 具有低色散; (2)在微波频段, 具有低介电常数, 容易实现光波与微波的相速匹配; (3)有机聚合物光 波导的加工工艺比硅基无机光波导简单、价格低廉、 无需高温加热, 只需通过室温旋涂和光刻等工艺就 可以制作出复杂的光集成器件; (4)有机聚合物光波 导和调制器、光开关等光电子器件的制备工艺与半导 体微电子器件的制备工艺一致, 这为光电集成的实 现打下基础. 基于以上的原因, 有机聚合物材料已经 受到越来越多的重视.

有机聚合物 $\mathrm{S}$ 形脊波导是有机聚合物光波导延 迟线的重要组成部分 ${ }^{[5]}$, 因此对它的优化设计具有非 常重要的意义. 本文将有效折射率法 ${ }^{[6]}$ 与变分法 ${ }^{[7,8]}$ 相结合, 分析了聚合物 $\mathrm{S}$ 形脊波导中基模和高阶模的 色散特性与场分布. 一般而言, 有效折射率法可以分 析光波导的色散特性, 计算量不大, 但是要准确计算 横向场的分布存在相当的难度, 采用变分法能够获 得较精确的光场分布. 研究表明, 将有效折射率法与
变分法相结合, 发挥了两种方法的长处, 既能获得准 确的横向场分布又能对色散特性进行分析. 然后利 用广角有限差分束传播法和有效折射率法研究了 $\mathrm{S}$ 形脊波导圆弧曲率半径与弯曲损耗的关系, 优化了 有机聚合物 S 形脊波导的结构参数.

\section{1 有机聚合物 $\mathrm{s}$ 形脊波导的结构}

有机聚合物 S 形脊波导如图 1 所示, 它由两段直 波导、两段 $1 / 4$ 圆弧波导与两段半圆弧波导组成, 半 圆弧波导比 $1 / 4$ 圆弧波导的曲率半径小, 以避免弯波 导与直波导的邻近耦合, $1 / 4$ 圆弧波导与直波导、 $1 / 4$

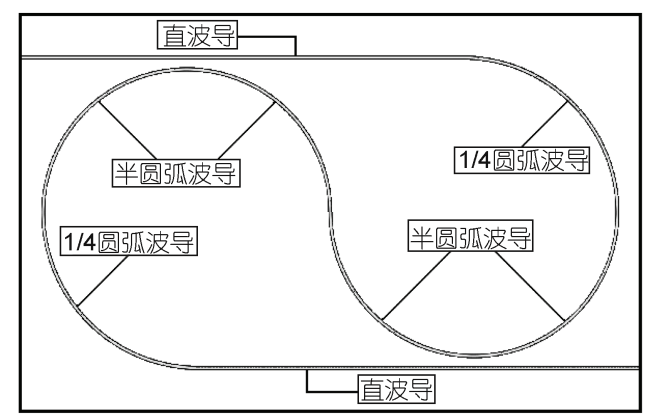

图 $1 \mathrm{~S}$ 形脊波导结构 
圆弧波导与半圆弧波导、半圆弧波导与半圆弧波导都 是相切连接. 横截面结构如图 2 所示, 上包层采用紫 外固化环氧 NOA61, 其折射率 $n_{l}=1.55$; 下包层采 用紫外固化环氧 UV15, 其折射率 $n_{3}=1.50$; 芯层是 由聚砜中掺人重量百分比 $25 \%$ 的生色团 ICP-E 构成, 折射率为 $n_{2}=1.67$.

\section{2 脊形光波导的色散特性和模场分布}

\section{1 变分有效折射率法推导}

脊形光波导的结构如图 2 所示, 脊的宽度为 $w$, 上、下包层的折射率分别为 $n_{1}$ 和 $n_{3}$, 芯层的折射率为 $n_{2}$; 芯层的厚度为 $d$, 脊处的厚度为 $D$. 在等效的平 板波导中, 分别在区域 I， II， III 中考虑 TE 和 TM 模, 利用有效折射率法得到如下本征方程：

$$
\gamma_{2} H=\operatorname{arctg}\left(c_{1} \frac{\gamma_{1}}{\gamma_{2}}\right)+\operatorname{arctg}\left(c_{2} \frac{\gamma_{3}}{\gamma_{2}}\right)+q \pi(q=0,1,2, \cdots),
$$

其中

$$
\begin{gathered}
\gamma_{1}=k_{0} \sqrt{n_{e f f i}^{2}-n_{1}^{2}}, \gamma_{2}=k_{0} \sqrt{n_{2}^{2}-n_{e f i}^{2}}, \gamma_{3}=k_{0} \sqrt{n_{e f i}^{2}-n_{3}^{2}}, \\
k_{0}=2 \pi / \lambda_{0},
\end{gathered}
$$

式中 $i=1,2,3$, 分别对应上述三个区域. 在区域 I 和 III 中, $H=d$, 在区域 II 中, $H=D$. 对于基模 $q=0, \lambda_{0}$ 为自由空间 工作波长. 对于 $\mathrm{TE}$ 模, $c_{1}=c_{2}=1$; 对于 $\mathrm{TM}$ 模, $c_{1}=n_{2}^{2} / n_{1}^{2}, c_{2}=n_{2}^{2} / n_{3}^{2}$. 求解本征方程(1)可得出三个区 域的有效折射率 $n_{\text {effi }}$, 即有效折射率的横向分布.

根据有效折射率的横向分布，考虑下述本征方 程

$$
\gamma_{2} w=\operatorname{arctg}\left(c_{1} \frac{\gamma_{1}}{\gamma_{2}}\right)+\operatorname{arctg}\left(c_{2} \frac{\gamma_{3}}{\gamma_{2}}\right)+p \pi(p=0,1,2, \cdots),
$$

其中

$$
\gamma_{1}=k_{0} \sqrt{n_{\mathrm{eff}}^{2}-n_{\mathrm{eff} 1}^{2}}, \gamma_{2}=k_{0} \sqrt{n_{\mathrm{eff} 2}^{2}-n_{\mathrm{eff}}^{2}}, \gamma_{3}=k_{0} \sqrt{n_{\mathrm{eff}}^{2}-n_{\mathrm{eff}}^{2} 3} .
$$

对于 $\mathrm{TE}$ 模, $c_{1}=n_{\mathrm{eff} 2}^{2} / n_{\mathrm{eff} 1}^{2}, c_{2}=n_{\mathrm{eff} 2}^{2} / n_{\mathrm{eff3}}^{2}$; 对于 $\mathrm{TM}$ 模, $c_{1}=c_{2}=1$. 求解方程 (4) 可得出脊形光波导中

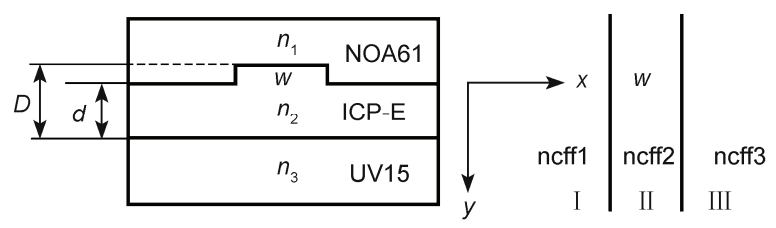

图 2 波导截面图和等效的平板波导
$\mathrm{TE}_{p q}$ 或 $\mathrm{TM}_{p q}$ 模的等效折射率 $n_{\mathrm{eff}}$, 其中 $p q$ 是模式标号. 如图 2 所示，由有效折射率法可得平板波导 $y$ 方 向近似的场分布函数，在区域 I 和 III 中:

$$
Y_{1}(y)=\left\{\begin{array}{lc}
\cos \left(\gamma_{2} d+\alpha\right) \exp \left[-\gamma_{1}(y-d)\right], & y>d, \\
\cos \left(\gamma_{2} y+\alpha\right), & 0<y<d, \\
\cos \alpha \exp \left(\gamma_{3} y\right), & y<0,
\end{array}\right.
$$

其中

$$
\begin{aligned}
\gamma_{1} & =k_{0} \sqrt{n_{\text {eff } 1}^{2}-n_{1}^{2}}, \gamma_{2}=k_{0} \sqrt{n_{2}^{2}-n_{\text {eff } 1}^{2}}, \gamma_{3} \\
& =k_{0} \sqrt{n_{\text {eff } 1}^{2}-n_{3}^{2}}, \\
\alpha & =-\operatorname{arctg}\left(c_{2} \frac{\gamma_{3}}{\gamma_{2}}\right)+q \pi(q=0,1,2, \ldots \ldots) .
\end{aligned}
$$

在区域 II 中:

$$
Y_{2}(y)=\left\{\begin{array}{lc}
\cos \left(\gamma_{2}^{\prime} D+\beta\right) \exp \left[-\gamma_{1}^{\prime}(y-D)\right], & y>D, \\
\cos \left(\gamma_{2}^{\prime} y+\beta\right), & 0<y<D,(9) \\
\cos \beta \exp \left(\gamma_{3}^{\prime} y\right), & y<0,
\end{array}\right.
$$

其中

$$
\begin{aligned}
\gamma_{1}^{\prime} & =k_{0} \sqrt{n_{\text {eff } 2}^{2}-n_{1}^{2}}, \gamma_{2}^{\prime}=k_{0} \sqrt{n_{2}^{2}-n_{\text {eff } 2}^{2}}, \gamma_{3}^{\prime} \\
& =k_{0} \sqrt{n_{\text {eff } 2}^{2}-n_{3}^{2}}, \\
\beta= & -\operatorname{arctg}\left(c_{2} \frac{\gamma_{3}^{\prime}}{\gamma_{2}^{\prime}}\right)+q \pi(q=0,1,2, \ldots \ldots .),
\end{aligned}
$$

在(8)与(11)式中, 对 TE模 $c_{2}=1$, 对 TM 模 $c_{2}=n_{2}^{2} / n_{3}^{2}$. 同样, 平板波导 $x$ 方向近似的场分布为:

$$
X(x)=\left\{\begin{array}{lr}
\cos \phi \exp \left[\gamma_{1}^{\prime \prime}(x+w / 2)\right], & x<-w / 2, \\
\cos \left(\gamma_{2}^{\prime \prime}(x+w / 2)+\phi\right), & -w / 2<x<w / 2, \\
\cos \left(\gamma_{2}^{\prime \prime} w+\phi\right) \exp \left[-\gamma_{3}^{\prime \prime}(x-w / 2)\right], x>w / 2,
\end{array}\right.
$$

式中

$$
\begin{aligned}
\gamma_{1}^{\prime \prime} & =k_{0} \sqrt{n_{\text {eff }}^{2}-n_{\text {eff } 1}^{2}}, \gamma_{2}^{\prime \prime}=k_{0} \sqrt{n_{\text {eff } 2}^{2}-n_{\text {eff }}^{2}}, \gamma_{3}^{\prime \prime} \\
& =k_{0} \sqrt{n_{\text {eff }}^{2}-n_{\text {eff } 3}^{2}}, \\
\phi & =-\operatorname{arctg}\left(c_{1} \frac{\gamma_{3}^{\prime \prime}}{\gamma_{2}^{\prime \prime}}\right)+p \pi(p=0,1,2, \ldots \ldots .),
\end{aligned}
$$

上式中 $c_{1}=n_{\mathrm{eff2}}^{2} / n_{\mathrm{effl}}^{2}$ 时为 TE 模, $c_{1}=1$ 时为 $\mathrm{TM}$ 模. 为得出较精确的光场分布, 考虑二维标量波动方程

$$
\frac{\partial^{2} \psi}{\partial x^{2}}+\frac{\partial^{2} \psi}{\partial y^{2}}+k_{0}^{2}\left[n^{2}(x, y)-n_{\mathrm{eff}}^{2}\right] \psi=0 .
$$

利用上述有效折射率法的结果，光波导中场分布 
$\psi(x, y)$ 可表示为子区域中场的叠加即:

$$
\psi(x, y)=X(x)\left[Y_{1}(y)+R Y_{2}(y)\right] .
$$

其中 $X(x) Y_{1}(y)$ 与 $X(x) Y_{2}(y)$ 分别是区域 I, III 和 II 中 的近似光场分布, $R$ 为待定的变分参量. 为确定变分 参量, 将(15)式两边乘以 $\psi$, 在横截面计算窗口内 积分:

$$
\begin{aligned}
& \iint\left[\frac{\partial^{2} \psi}{\partial x^{2}}+\frac{\partial^{2} \psi}{\partial y^{2}}\right] \psi \mathrm{d} x \mathrm{~d} y+k_{0}^{2} \iint n^{2} \psi^{2} \mathrm{~d} x \mathrm{~d} y \\
& -k_{0}^{2} n_{\mathrm{eff}}^{2} \iint \psi^{2} \mathrm{~d} x \mathrm{~d} y=0 .
\end{aligned}
$$

将(16)式代人(17)式中经整理得到如下的二次代数 方程:

$$
a R^{2}+b R+c=0,
$$

其中

$$
\begin{aligned}
a= & \iint\left(X X^{\prime \prime} Y_{2}^{2}+X^{2} Y_{2} Y_{2} d \varnothing\right) \mathrm{d} x \mathrm{~d} y \\
& +k_{0}^{2} \iint n^{2} X^{2} Y_{2}^{2} \mathrm{~d} x \mathrm{~d} y-k_{0}^{2} n_{e f f}^{2} \iint X^{2} Y_{2}^{2} \mathrm{~d} x \mathrm{~d} y, \\
b= & \frac{1}{2} \iint\left(X X^{\prime \prime} Y_{1} Y_{2}+X^{2} Y_{1}^{\prime \prime} Y_{2}\right) \mathrm{d} x \mathrm{~d} y \\
& +\frac{1}{2} \iint\left(X X^{\prime \prime} Y_{1} Y_{2}+X^{2} Y_{1} Y_{2}^{\prime \prime}\right) \mathrm{d} x \mathrm{~d} y \\
& +k_{0}^{2} \iint n^{2} X^{2} Y_{1} Y_{2} \mathrm{~d} x \mathrm{~d} y-k_{0}^{2} n_{e f f}^{2} \iint X^{2} Y_{1} Y_{2} \mathrm{~d} x \mathrm{~d} y, \\
c= & \iint\left(X X^{\prime \prime} Y_{1}^{2}+X^{2} Y_{1}^{\prime \prime} Y_{1}\right) \mathrm{d} x \mathrm{~d} y \\
& +k_{0}^{2} \iint n^{2} X^{2} Y_{1}^{2} \mathrm{~d} x \mathrm{~d} y-k_{0}^{2} n_{e f f}^{2} \iint X^{2} Y_{1}^{2} \mathrm{~d} x \mathrm{~d} y,
\end{aligned}
$$

把 $a, b, c$ 后代回(18)式可以解出变分参量 $R$, 计算表明 变分参量有两个解, 必须取 $|R|$ 较小的一个, 由(16)式 即可得出光波场的横向分布.

\section{2 脊形波导中 TE 和 TM 模的色散特性分析}

脊形波导结构如图 2 所示, $w$ 是脊形波导中脊的 宽度, 脊的高度取为 $0.3 \mu \mathrm{m}$. 利用上述理论对 TE, TM 模分别进行分析, 取脊宽的变化范围为 $3 \sim 9 \mu \mathrm{m}$, 步 长 $\Delta w=1 \mu \mathrm{m}$, 其有效折射率随脊宽的变化曲线如图 3 所示.

工作波长的变化范围取为 $1.0 \sim 1.7 \mu \mathrm{m}$, 步长 $\Delta \lambda$ $=0.1 \mu \mathrm{m}, \mathrm{TE}$ 和 $\mathrm{TM}$ 基模等效折射率随波长的变化曲 线如图 4 所示：从图中可以看出等效折射率随波长的 增加而单调下降.
脊高的变化范围取为 $0.1 \sim 1.0 \mu \mathrm{m}$, 步长 $\Delta h=$ $0.1 \mu \mathrm{m}, \mathrm{TE}, \mathrm{TM}$ 基模等效折射率随脊高的变化曲线 如图 5 所示：由图可见基模等效折射率随脊高的增加 而单调增加.

从上述计算结果可以看出，聚合物脊形光波导 具有下列一些特点: (1)有效折射率随脊宽的变化较 缓慢，随着模式阶数的增加有效折射率明显降低; (2) 等效折射率随波长的增加单调下降，随脊高的增加 单调上升; (3)在结构参数与工作波长相同的情况下, 同一阶 TM 模的有效折射率较 TE 模的有效折射率要 小. 对多模光波导而言，高阶模的传输条件对应于 相应的本征方程具有实解, 由此可以得到：当芯层 厚度与脊宽分别满足 $d \leqslant 1.5 \mu \mathrm{m}, w \leqslant 5 \mu \mathrm{m}$ 时，聚合物
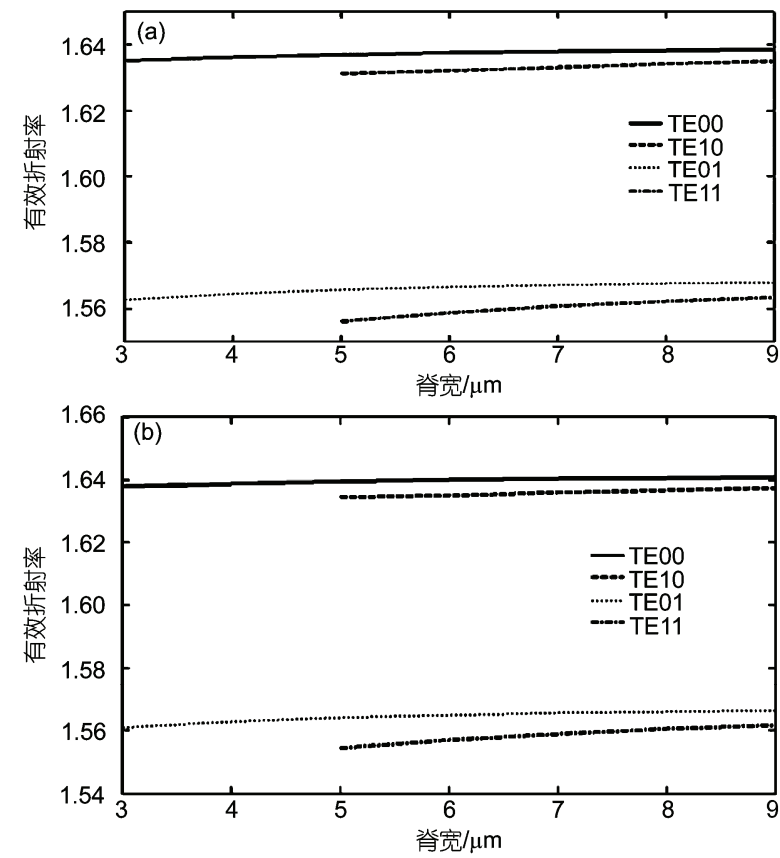

图 3 有效折射率随脊宽的变化曲线 (a)TM 模; (b)TE 模

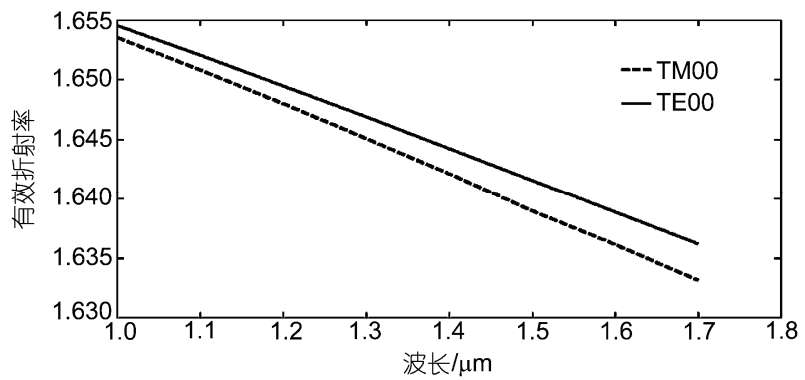

图 4 基模有效折射率随工作波长的变化曲线 


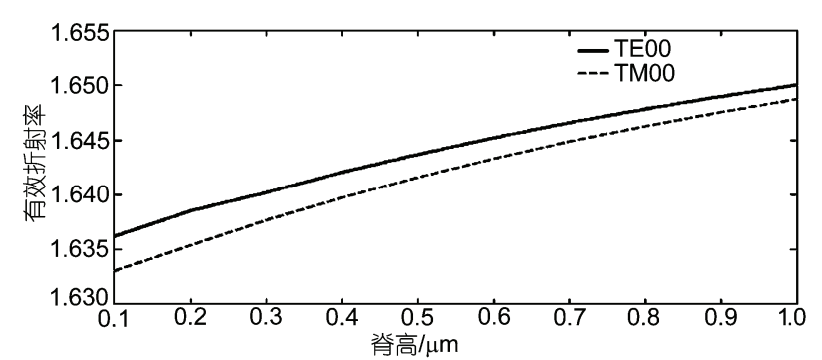

图 5 基模有效折射率随脊高的变化曲线

光波导实现单模传输.

\subsection{TM 模的横向光场分布}

应用变分有效折射率法,对脊形光波导不同波导 结构参数计算出其光场横向场分布, 对应的光强分 布如图 6 和 7 所示. 图 6 波导结构参数为 $D=1.8 \mu \mathrm{m}$, $d=1.5 \mu \mathrm{m}, w=5 \mu \mathrm{m}$, 图 7 波导结构参数为 $D=1.9 \mu \mathrm{m}$, $d=1.6 \mu \mathrm{m}, w=6 \mu \mathrm{m}$, 工作波长为 $\lambda_{0}=1.55 \mu \mathrm{m}$.

\section{3 聚合物 $\mathrm{S}$ 形脊波导圆弧曲率半径与弯曲 损耗的关系}

\section{1 广角 FD-BPM 与 Padé 近似}

本文所设计的 $\mathrm{S}$ 形光波导中的光场传播不满足

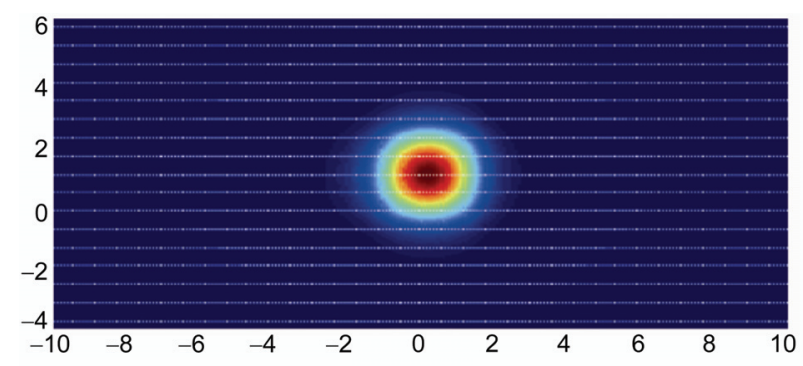

图 $6 \mathrm{TMO0}$ 模的横向场分布

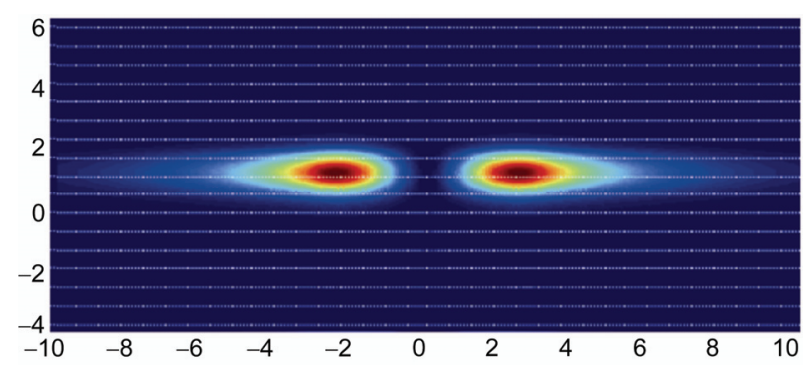

图 7 TM10 模的横向场分布
近轴条件, 需要用广角 FD-BPM ${ }^{[9]}$ 来分析计算.

在弱导条件下，可以得到亥姆霍兹(Helmholtz) 方程:

$$
\nabla^{2} E+n^{2} k_{0} E=0,
$$

式中, $E(x, y, z)$ 是电场分布, $n(x, y, z)$ 是波导折射率分布, $K_{0}$ 为真空中的波数, $k_{r}$ 通常取值等于 $k_{0} \times n$ 褯, 令

$$
E(x, y, z)=u(x, y, z) \exp (i \omega t) \exp \left(i k_{r} z\right),
$$

式中 $\omega$ 为光频, $k_{r}$ 为任意常数. 于是标量 Helmholz 方程为:

$$
u_{z z}+2 i k_{r} u_{z}+u_{x x}+\left(k^{2}-k_{r}^{2}\right) u=0,
$$

将(24)式写成

$$
u_{z}=i k_{r}(\sqrt{1+P}-1) u .
$$

算子 $P=\left(\partial^{2} / \partial x^{2}+\left(k^{2}-k_{r}^{2}\right)\right) / k_{r}^{2}$, 根据 Padé 近似 ${ }^{[9]}$, $\sqrt{1+P}-1 \approx N_{m}(P) / D_{n}(P)$, 其中 $N_{m}$ 和 $D_{n}$ 分别为算子 $P$ 的 $m$ 和 $n$ 阶多项式, 把(25)式改写为

$$
u_{z}=i k_{r}\left(N_{m}(P) / D_{n}(P)\right) u \text {. }
$$

为精确模拟波导中光场特性, 对(26)式采用二阶 Padé近似

$$
u_{z}=i k_{r} \frac{P / 2+P^{2} / 4}{1+3 P / 4+P^{2} / 16} u,
$$

对(27)式在点 $(s, r+1 / 2)$ ( $s$ 为 $x$ 方向, $r$ 为 $z$ 方向)进行 离散化, 先在 $z$ 轴方向离散, 取 $u_{z}=\left(u^{r+1}-u^{r}\right) / \Delta z$, $u=\left(u^{r+1}+u^{r}\right) / 2$, 可得

$$
u^{r+1}=\frac{1+\xi_{1} P+\xi_{2} P^{2}}{1+\eta_{1} P+\eta_{2} P^{2}} u^{r},
$$

其中 $\xi_{1}=3 / 4+i k_{r} \Delta z / 4, \quad \xi_{2}=1 / 16+i k_{r} \Delta z / 8, \quad \eta_{1}=3 / 4$ $-i k_{r} \Delta z / 4, \eta_{2}=1 / 16-i k_{r} \Delta z / 8,(28)$ 式可以写成

$$
u^{r+1}=\frac{\left(1+a_{1} P\right)\left(1+a_{2} P\right)}{\left(1+b_{1} P\right)\left(1+b_{2} P\right)} u^{r},
$$

其中 $a_{i}, b_{i}(i=1,2)$ 可以通过多项式方程求解得到, 将 (29)式分为两步运算, 每一步的形式为

$$
u^{r+i / 2}=\frac{\left(1+a_{i} P\right)}{\left(1+b_{i} P\right)} u^{r+(i-1) / 2},
$$

把(30)式在 $x$ 轴方向离散, 取 $\partial^{2} u / \partial x^{2}=\left(u_{s+1}-2 u_{s}\right.$ $\left.+u_{s-1}\right) / \Delta x^{2}$, 可得

$$
A \mathrm{u}_{s-1}^{r+i / 2}+B \mathrm{u}_{s}^{r+i / 2}+C \mathrm{u}_{s+1}^{r+i / 2}=D,
$$


其中

$$
\left.\begin{array}{rl}
A= & C=\frac{b_{i}}{\Delta x^{2} k_{r}^{2}} \\
B & =1+\frac{b_{i}}{k_{r}^{2}}\left[\left(k^{2}-k_{r}^{2}\right)-\frac{2}{\Delta x^{2}}\right] \\
D & =\frac{a_{i}}{\Delta x^{2} k_{r}^{2}}\left(u_{s-1}^{r+(i-1) / 2}+u_{s+1}^{r+(i-1) / 2}\right) \\
& \left.+\left\{1+\frac{a_{i}}{k_{r}^{2}}\left[\left(k^{2}-k_{r}^{2}\right)-\frac{2}{\Delta x^{2}}\right]\right\} u_{s}^{r+(i-1) / 2}\right\}
\end{array}\right\}
$$

这样, 如果已知前一步的场量 $u^{r}$, 便可以应用追 赶法通过两步计算得到下一步的场量 $u^{r+1}$.

\section{2 有效折射率的确定}

如图 2 所示, 取脊波导的芯层为 $1.5 \mu \mathrm{m}$, 脊高为 $0.3 \mu \mathrm{m}$, 脊宽为 $5 \mu \mathrm{m}$, 上下包层的厚度分别为 $3.5 \mu \mathrm{m}$ 和 $3 \mu \mathrm{m}$. 求解本征方程(1)可得到三个区域的有效折 射率 $n_{\text {eff }}$. 通过计算得到脊形波导中 TM 基模的有效 折射率横向分布为:

$$
n(x)=\left\{\begin{array}{l}
n_{\text {eff } 1},|x| \leqslant w / 2, \\
n_{\text {eff } 2},|x|>w / 2,
\end{array}\right.
$$

其中 $n_{\text {eff } 1}=1.6373, n_{\text {eff } 2}=1.6313, \Delta n=0.006$.

\section{3 仿真分析}

在 $\mathrm{S}$ 形波导器件中, 波导弯曲半径越小, 器件结 构越紧凑, 而由此引起的损耗会越大. 波导发生弯曲 时, 不考虑波导的结构缺陷等引人的损耗, 仅基于弯 曲的本身考虑, 可以分成两部分: 纯弯曲损耗和过渡 损耗. 前者是指具有连续弯曲形状变化和连续弯曲 曲率变化的弯曲波导中, 由于光波传播方向的改变 而引起的光波损耗, 当波导弯曲时, 光场峰值向着远 离圆弧中心方向发生偏移, 光场因而变得不对称, 因 为弯曲模为泄漏模, 光功率切向离开波导而转化成 辐射模形成辐射损耗; 后者是由于在弯曲时, 波导曲 率变化的不连续所引起的损耗, 通常这部分损耗会 发生在直波导与弯曲波导之间的连接处 ${ }^{[10]}$.

接下来, 我们研究 $\mathrm{S}$ 形波导圆弧部分的曲率半径 对弯曲损耗的影响. 在以下的分析中, 一些统一参数 设置如下：激励光源波长为 $1.55 \mu \mathrm{m}$, 计算步长 $\Delta x=\Delta z=0.5 \mu \mathrm{m}$, 激励光源为双曲余弦形.

我们设定计算窗口大小为 900 (步)×2000(步), 分
别对圆弧半径为 $400,1000,2000$ 和 $5000 \mu \mathrm{m}$ 的圆弧 波导进行了导光仿真, 图 8 10 为半径为 400, 1000, 2000 和 $5000 \mu \mathrm{m}$ 的波导中的能量弯曲损耗情形: 在 半径为 $400 \mu \mathrm{m}$ 时, 弯曲损耗很大, 光能量在波导中 传播约 $300 \mu \mathrm{m}$ 后便辐射殆尽; 半径为 $1000 \mu \mathrm{m}$ 时, 大部分光能量可以在波导中稳定传播较长的距离(约 $700 \mu \mathrm{m})$; 半径为 2000 和 $5000 \mu \mathrm{m}$ 时, 光能量在计算
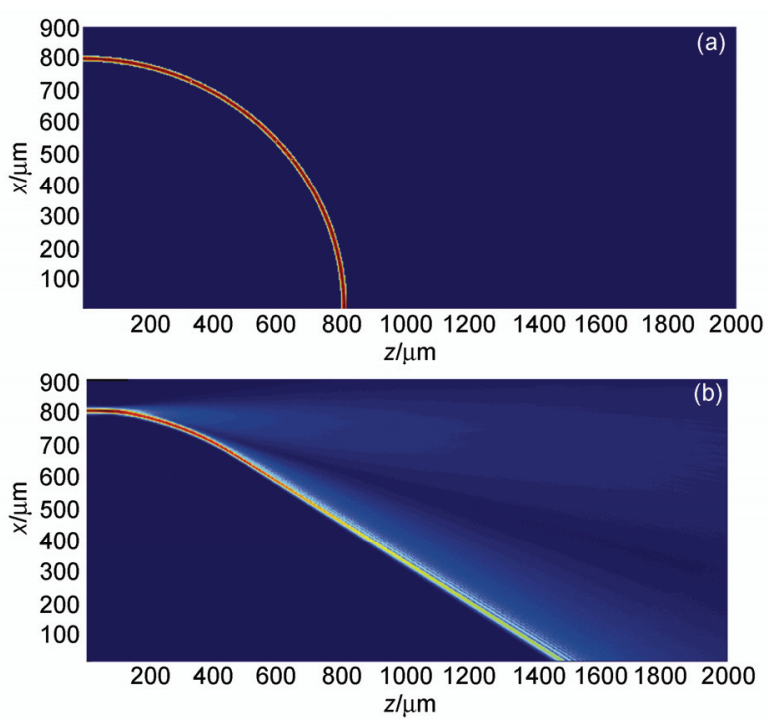

图 8 半径 $400 \mu \mathrm{m}$ 的圆弧波导中的光能量弯曲损耗情形 (a)波导形状; (b)光场
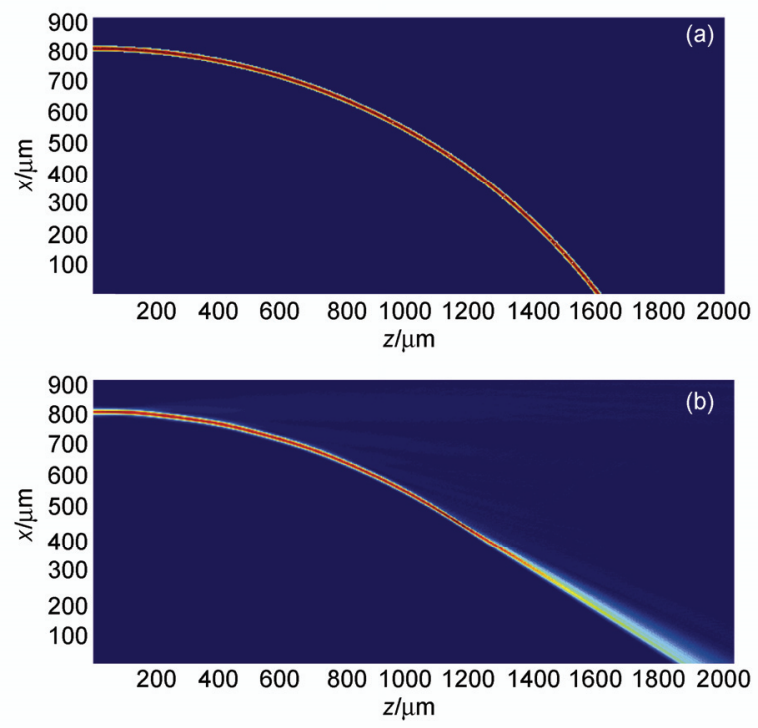

图 9 半径 $1000 \mu \mathrm{m}$ 的圆弧波导中的光能量弯曲损耗情形 (a)波导形状; (b)光场 

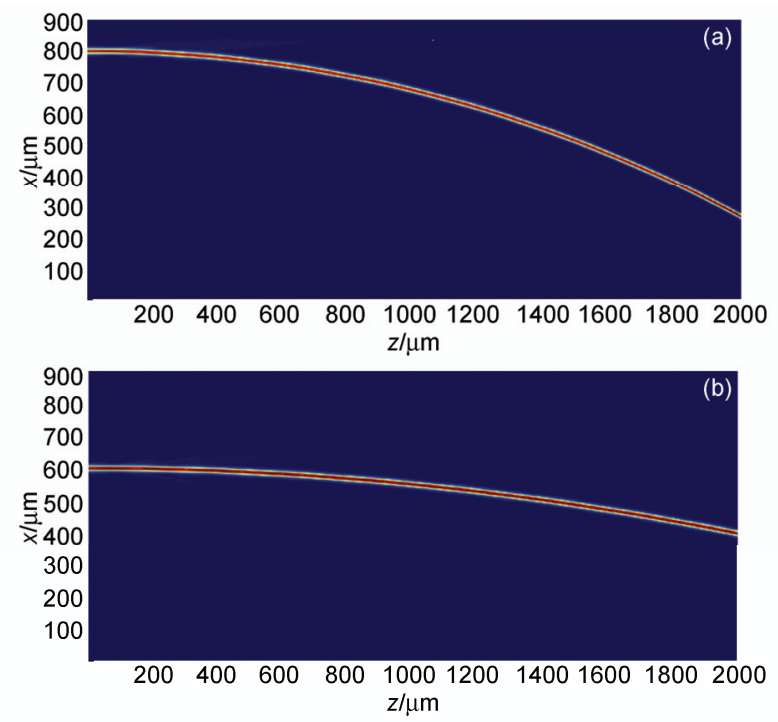

图 10 半径 2000 和 $5000 \mu \mathrm{m}$ 的圆弧波导中光能量弯曲损耗 情形

(a) $2000 \mu \mathrm{m}$; (b) $5000 \mu \mathrm{m}$

窗口所确定的长度范围内没有出现大量的辐射损耗. 由此可知, 随着圆弧波导曲率半径的增大, 波导的弯 曲辐射损耗越来越小, 光能量可以越来越稳定地在 波导中传输.

图 11 是归一化平均辐射能量与波导曲率半径的关 系曲线, 观察可知, 当半径大于 $5000 \mu \mathrm{m}$ 后, 曲线开 始变得非常平缓, 这说明此后随着波导曲率半径的 增大, 波导的弯曲辐射损耗已不会再明显减小, 光能 量已可以在 $\mathrm{S}$ 形波导中稳定的传播, 形成导模, 这

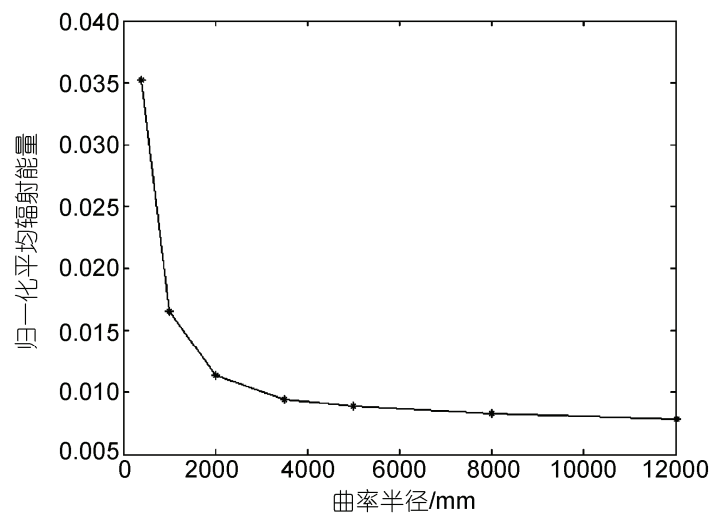

图 11 归一化平均辐射能量与波导曲率半径的关系

一点是很有参考价值的.

\section{4 结论}

本文利用变分有效折射率法对聚合物脊形多模 光波导基模和高阶模的色散特性与横向场分布进行 分析, 研究了波导结构参数对色散特性的影响, 计算 出 TM 基模和高阶模的光场分布, 得出了聚合物脊形 光波导的单模传输条件: 芯层厚度与脊宽分别满足 $d \leqslant 1.5 \mu \mathrm{m} ， w \leqslant 5 \mu \mathrm{m}$. 利用广角有限差分束传播 法和有效折射率法研究了 S 形脊波导圆弧曲率半径与 弯曲损耗的关系，得出了如下结论：当圆弧曲率半径 大于 $5000 \mu \mathrm{m}$ 后，即使波导曲率半径增大，波导的弯 曲辐射损耗已不会再明显减小, 光已可以在 S 形脊波 导中稳定地传播了.

\section{参考文献}

1 Robert A N, Robert B, Louay A E,et al. Polymer integrated optical devices for telecommunications applications. Proc SPIE, 1998, 3282: $2-13$

2 Yao H H, Norbert K, Crispin Z, et al. polymeric planar waveguide devices for photonic network applications. Proc SPIE, 2001, 4439: $36-50$

3 Yutaka O,Hirotake K,Takahisa T, et al. Integration of organic electroluminescent diodes and polymeric waveguide devices: Characterization of light source for optical integrated circuit. Proc SPIE, 2001, 4439: 95-102

4 Kang J-W, Eunkyoung K, Kim M J, et al. All-optical polymer waveguide devices. Proc SPIE, 2002, 4905: 108_-117

5 James F, Jennifer C, Tang Y J, et al. Switched optical polymeric waveguide true-time-delay lines for wideband photonics phased array antennas. Proc SPIE, 2004, 5356: 65-73

6 Winick K A. Effective-index method and coupled-mode theory for almost-periodic waveguide gratings: A comparison. Appli Opt, 1992, 31: $757-761$

7 张雅丽，张蹈，祝宁华. 掩埋型离子交换玻璃光波导的变分分析. 光学学报, 2007, 27: 981一986

8 Weiping Huang, Hermann A.Huas. A simple variational approach to optical rib waveguides. IEEE J Light Tech, 1991, 9: 56-61

9 Kenji K, Tsutomu K. Introduction to optical waveguide analysis. New York: Wiley-Interscience, 2001. 204-213

10 杨建义, 王明华. 光波导弯曲的结构优化. 光电子·激光, 1999, 10: 305-309 